\title{
Robust Model Predictive Control under Redundant Channel Transmission with Applications in Networked DC Motor Systems
}

\author{
Yan Song, Zidong Wang, Derui Ding and Guoliang Wei
}

\begin{abstract}
In networked systems, intermittent failures in data transmission are usually inevitable due to the limited bandwidth of the communication channel, and an effective countermeasure is to add redundance so as to improve the reliability of the communication service. This paper is concerned with the model predictive control (MPC) problem by using static output feedback for a class of polytopic uncertain systems with redundant channels under both input and output constraints. By utilizing the min-max control approach combined with stochastic analysis, sufficient conditions are established to guarantee the feasibility of the designed MPC scheme that ensures the robust stability of the closed-loop system. In terms of the solution to an auxiliary optimization problem, an easy-to-implement MPC algorithm is proposed to obtain the desired sub-optimal control sequence as well as the upper bound of the quadratic cost function. Finally, to illustrate its effectiveness, the proposed design method is applied to control a networked direct current (DC) motor system.
\end{abstract}

Index Terms-Model predictive control, Networked control systems, Static output feedback, Redundant channels, DC motor control, Equality constraints.

\section{INTRODUCTION}

$\mathbf{M}$ ODEL predictive control (MPC), also called receding horizon control, has been widely applied into the process industries in the past few decades due mainly to its ability to handle input/state constraints and compensate time delays [18], [19], [26]. Different from the conventional control with a pre-computed control law, MPC requires online optimization to compute an optimal control sequence at every sampling interval and implements the first one of the control sequence. Recently, with the ever-increasing popularity of fast micro-controllers, MPC has also become a very powerful and attractive alternative to classical controllers in the areas of power converters and electrical drives, see [2], [12], [14], [21], [25], [28] and the references therein. For instance, an

This work was supported in part by the Royal Society of the UK, the National Natural Science Foundation of China under Grants 61329301, 61403254, 61374039 and 61304095, Shanghai Pujiang Program under Grant 13PJ1406300, Shanghai Natural Science Foundation of China under Grant 13ZR1428500, Innovation Program of Shanghai Municipal Education Commission under Grant 14YZ083, the Hujiang Foundation of China under Grants C14002 and D15009, and the Alexander von Humboldt Foundation of Germany.

Y. Song, D. Ding and G. Wei are with the Shanghai Key Lab of Modern Optical System, Department of Control Science and Engineering, University of Shanghai for Science and Technology, Shanghai 200093, China. (Email: sonyalusst.edu.cn)

Z. Wang is with the Department of Computer Science, Brunel University London, Uxbridge, Middlesex, UB8 3PH, United Kingdom. He is also with the Faculty of Engineering, King Abdulaziz University, Jeddah 21589, Saudi Arabia. (Email: Zidong.Wang@brunel.ac.uk)
MPC-based voltage control issue has been addressed in [12] to restore voltage following a contingency and maintain a prespecified amount of post-transient voltage stability margin. In [21], an MPC-based maximum power point tracking strategy has been presented, in which the predictive controller could correct errors before the switching signal was applied to the respective converter.

It should be pointed out that the utilization of the MPC strategy does not inherently ensure the closed-loop stability. For example, the aforementioned MPC algorithms might not be able to guarantee the robustness against model uncertainties. Accordingly, the design of a robust and stable MPC algorithm for uncertain system with constraints has become an ongoing research issue that attracts increasing attention from researchers. Some representative results on this issue can be highlighted as follows. In [10], a direct digital MPC controller design method without approximation has been proposed for the continuous-time systems with saturation constraints. Very recently, some sufficient conditions ensuring the feasibility and stability have been investigated in [16] for nonlinear systems subject to bounded disturbances, and then an event-triggered MPC algorithm has been provided in order to efficiently exploit system resources. For a class of distributed nonlinear systems, the feasibility and the stability of the robust distributed MPC scheme have been discussed in [17] by utilizing the triangle inequality and the GronwallBellman inequality.

Note that, in most previous works on MPC, it has been implicitly assumed that system states are fully accessible. This is, unfortunately, not always the case in practice, and it is often necessary to design the output feedback controller in the framework of MPC to satisfy specified performance such as stability and robustness. However, so far, only limited research results have been available in the literature for uncertain systems (see e.g. [4], [15]) due mainly to the difficulty of finding an appropriate methodology to optimize the quadratic cost performance with incomplete measurement information in comparison with the traditional optimal control approaches.

In networked systems, the limited bandwidth of the communication channel inevitably gives rise to various networkinduced phenomena such as the transmission delay [24], missing measurements (or packet dropouts), signal quantization, and so forth. It is worth mentioning that, if not properly handled, these network-induced phenomena (especially missing measurements) could cause performance degradation of the underlying systems. To ensure the quality and reliability of 
the communication service, a new communication protocol, namely, redundant channel transmission, has received particular research attention in recent years. Such a strategy has been widely utilized in some industrial systems and critical infrastructures such as power systems and aerospace fields.

In redundant channel schemes, if a channel suffers certain communication failure which can be detected by means of software or hardware devices, another channel will be automatically activated to protect the key data. In [20], a new hybrid adaptive modulation and diversity scheme, in which the same information has been transmitted redundantly over a subset of available sub-channels, has been provided to increase the overall spectral efficiency. In [29], by injecting artificial redundancy into wireless transmission, the transmission reliability has been enhanced. When it comes to networked control systems, the overall performance will be improved by using redundant channels as compared with single channel case, see [1], [27] for more details. Unfortunately, to the best of the authors' knowledge, such a communication protocol for MPC issues with input and output constraints has not been adequately investigated, not to mention the case when the output feedback control scheme is applied as well. It is, therefore, the purpose of this paper to shorten such a gap.

Motivated by the above discussions, in this paper, we aim to investigate the static output feedback MPC problem for a class of polytopic uncertain systems with input and output constraints under redundant channels. The main contributions of this paper can be highlighted as follows. 1) By utilizing a set of Bernoulli distributed random variables, a new measurement model is presented to describe the redundant channel communication protocol. 2) According to the min-max control approach, some sufficient conditions are proposed to verify the feasibility of the designed MPC scheme and the robust stability of the closed-loop system where the singular value decomposition approach is utilized to handle equality constraints. 3) An easy-to-implement MPC algorithm is provided to obtain the desired sub-optimal control sequence and derive an upper bound of certain quadratic cost function. 4) The proposed MPC scheme is applied into a direct current (DC) motor system in order to illustrate its effectiveness.

The rest of this paper is organized as follows. In Section II, a class of polytopic uncertain discrete-time linear systems with $N$ redundant channels and input/output constraints are presented. In Section III, for all admissible parameter uncertainties and packet dropouts, sufficient conditions are provided to guarantee the stability of the addressed systems and the feasibility of the given MPC scheme. Furthermore, by means of the solution to an optimization problem, an easyto-use MPC algorithm is proposed to obtain the desired suboptimal control sequence and also derive an upper bound of certain quadratic cost function. In Section IV, an example of direct current (DC) motor systems is utilized to demonstrate the effectiveness of the main results. Finally, conclusions are drawn in Section V.

Notation The notation used here is fairly standard except where otherwise stated. $\mathbb{R}^{n}$ and $\mathbb{R}^{n \times m}$ denote, respectively, the $n$-dimensional Euclidean space and the set of all $n \times m$ real matrices. $x(k+n \mid k), u(k+n \mid k)$ and $y(k+n \mid k)$ are the values of $x, u$ and $y$ at a future time $k+n .|x|$ and $\|x\|^{2}$ stand for the absolute value of the scalar $x$ and the Euclidean norm of the vector $x$, respectively. The notation $X \geq Y$ (respectively, $X>Y$ ), where $X$ and $Y$ are symmetric matrices, means that $X-Y$ is positive semi-definite (respectively, positive definite). $\|x\|_{W}^{2}=x^{T} W x$ where $W>0$ is a symmetric weighting matrix. $I$ and 0 denote the identity matrix and the zero matrix with appropriate dimensions, respectively. The superscripts " -1 " and " $T$ " are, respectively, the inverse and transpose of a matrix. $\mathbb{E}\{x\}$ stands for the expectation of the stochastic variable $x$. The symbol " $*$ " in a matrix denotes the symmetric part in a symmetric matrix. $\operatorname{diag}\{\cdots\}$ stands for a block-diagonal matrix.

\section{Problem Statement And PREliminaries}

\section{A. Problem Statement}

Consider the following polytopic uncertain discrete-time linear system with $N$ redundant channels:

$$
\left\{\begin{aligned}
x(k+1)= & A(k) x(k)+B(k) u(k) \\
y(k)= & \alpha_{1}(k) C_{1}(k) x(k) \\
& +\sum_{i=2}^{N}\left\{\prod_{j=1}^{i-1}\left(1-\alpha_{j}(k)\right) \alpha_{i}(k) C_{i}(k) x(k)\right\}
\end{aligned}\right.
$$

where $x(k) \in \mathbb{R}^{n_{x}}, u(k) \in \mathbb{R}^{n_{u}}$ and $y(k) \in \mathbb{R}^{n_{y}}$ are the state vector, the control input and the measured output, respectively. $A(k), B(k)$ and $C_{i}(k)(i=1,2 \cdots, N)$ with appropriate dimensions are unknown matrices which contain polytopic uncertainties (see e.g., [22]) given as follows:

$$
\Xi(k):=\left(A(k), B(k), C_{1}(k), \ldots, C_{i}(k), \ldots, C_{N}(k)\right) \in \Omega
$$

where $\Omega$ is a given convex bounded polyhedral domain described by

$$
\Omega:=\left\{\Xi(k) \mid \Xi(k)=\sum_{l=1}^{L} \lambda_{l} \Xi^{(l)}(k), \sum_{l=1}^{L} \lambda_{l}=1, \lambda_{l} \geq 0\right\}
$$

with known matrices $\Xi^{(l)}:=\left(A^{(l)}, B^{(l)}, C_{1}^{(l)}, \ldots, C_{N}^{(l)}\right)(l=$ $1,2, \ldots, L)$.

The random variables $\alpha_{i}(k)(i=1,2, \ldots, N)$, describing the packet dropout phenomena of the $i$ th channel, take values on 0 or 1 with

$$
\operatorname{Prob}\left\{\alpha_{i}(k)=1\right\}=\tilde{\alpha}_{i}, \operatorname{Prob}\left\{\alpha_{i}(k)=0\right\}=1-\tilde{\alpha}_{i}
$$

where $\tilde{\alpha}_{i}(i=1,2, \ldots, N)$ are the known non-negative constants. It is assumed that all the random variables $\alpha_{i}(k)$ are mutually independent.

Remark 1: As described in Fig. 1, an $N$ redundant channel transmission protocol is utilized with hope to improve the reliability of transmitted information. According to the measurement model in (1), if no packet dropout occurs at the first channel (i.e. $\alpha_{1}(k)=1$ ), the measurement output is described as $y(k)=C_{1}(k) x(k)$, which implies that the signal transmitted by other channels is not used. When $\alpha_{j}(k)=0$ $(j=1,2, \cdots, i-1)$ and $\alpha_{i}(k)=1$, that is, the channels from 1 to $i-1$ suffer from packet dropouts at time $k$, the MPC scheme will utilize the data transmitted by channel $i$ 


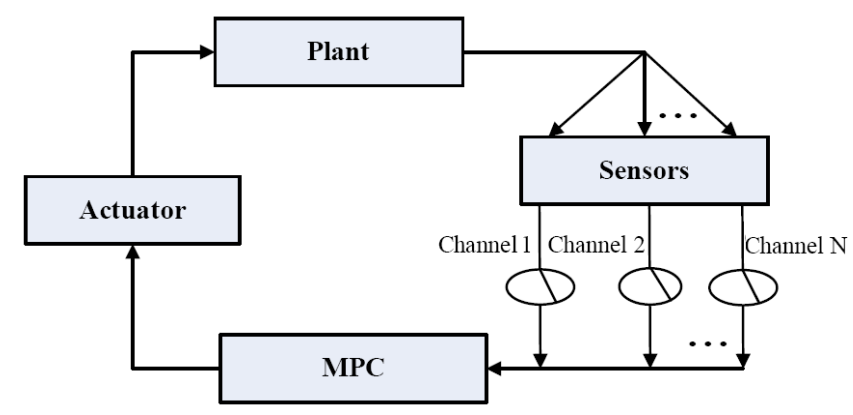

Fig. 1. The structure of the MPC system with redundant channels.

to execute the control tasks. Furthermore, when $\alpha_{j}(k)=0$ for $j=1,2, \cdots, N$, no information would be received by controllers. Obviously, the more redundant channels are applied in the networked communication, the more reliability will be guaranteed. On the other hand, the implementation of such a protocol needs some additional detection devices at each channel terminal to check whether the packet loss occurs. Therefore, there is a trade-off between the cost of equipment/energy and the quality of service.

In this paper, for the polytopic uncertain discrete-time linear system (1), we consider the following static output feedback (SOF) controller in the framework of MPC

$$
u(k+n \mid k)=F(k) y(k+n \mid k), \quad n=0,1,2, \ldots
$$

where $u(k+n \mid k)$ and $y(k+n \mid k)$ are the $n$-th step prediction of control input and measurement output at time $k, F(k)$ is the feedback gain to be designed. For simplicity, we denote $x(k \mid k)=x(k), u(k \mid k)=u(k)$ and $y(k \mid k)=y(k)$. In order to reflect the actual requirement of practical engineering, the constraints on control input and measurement output for discrete-time linear system (1) are given by

$$
\left\{\begin{array}{l}
\left|[u(k+n \mid k)]_{p}\right| \leq \bar{u}_{p}, \quad n \geq 0, p=1,2, \ldots, n_{u} \\
\left|[y(k+n \mid k)]_{q}\right| \leq \bar{y}_{q}, \quad n \geq 1, q=1,2, \ldots, n_{y}
\end{array}\right.
$$

where $[\cdot]_{p}\left([\cdot]_{q}\right)$ denotes the $p$ th $(q$ th $)$ element of a vector or the $p$ th $(q$ th) row of a matrix.

According to the principle of MPC, the closed-loop system can be obtained from (1) and (3) as follows

$$
x(k+1)=[A(k)+B(k) F(k) \Gamma(k)] x(k)
$$

where

$$
\Gamma(k)=\alpha_{1}(k) C_{1}(k)+\sum_{i=2}^{N}\left\{\prod_{j=1}^{i-1}\left(1-\alpha_{j}(k)\right) \alpha_{i}(k) C_{i}(k)\right\} .
$$

Furthermore, associated with the above closed-loop system, construct a min-max optimal cost of the form

$$
J(k):=\min _{F(k)} \max _{A^{(l)}, B^{(l)}, C_{1}^{(l)} \ldots C_{N}^{(1)} \in \Omega} \tilde{J}(k, x(k))
$$

where

$$
\tilde{J}(k, x(k))=\mathbb{E}\left\{\sum_{n=0}^{\infty}\left[\|x(k+n \mid k)\|_{Q}^{2}+\|u(k+n \mid k)\|_{R}^{2}\right]\right\}
$$

with given symmetric positive-definite weighting matrices $Q$ and $R$.

Our aim in this paper is to develop techniques to deal with the SOF MPC issue for polytopic uncertain discrete-time linear system (1) with $N$ redundant channels. More specifically, we like to design a controller of the form (3) for the system (1) with control and output constraints such that, for all admissible parameter uncertainties and packet dropouts, the following two requirements are satisfied simultaneously:

R1) the closed-loop system (5) is asymptotically stable in mean-square sense;

R2) an upper bound is obtained by solving the given minmax optimization problem at each time $k$.

\section{B. Preliminaries}

For the convenience of the later discussion, we give some important definitions and lemmas as follows.

Definition 1: The set

$$
\Pi(P(k), \gamma(k))=\left\{z \in \mathbb{R}^{n_{x}}: \mathbb{E}\left\{z^{T} P(k) z\right\} \leq \gamma(k)\right\}
$$

is called an ellipsoid in mean-square sense, where $P(k) \in$ $\mathbb{R}^{n_{x} \times n_{x}}$ is a positive definite matrix and $\gamma(k) \in \mathbb{R}$ is a positive scalar.

Lemma 1: [11] For any symmetric positive definite matrix $P \in \mathbb{R}^{n_{x} \times n_{x}}$, the map $\varrho \rightarrow \varrho^{T} P \varrho$ is convex, i.e.

$$
\left(\sum_{i=1}^{n} \delta_{i} \varrho_{i}\right)^{T} P\left(\sum_{i=1}^{n} \delta_{i} \varrho_{i}\right) \leq \sum_{i=1}^{n} \delta_{i} \varrho_{i}^{T} P \varrho_{i}, \quad \forall \varrho_{i} \in \mathbb{R}^{n_{x}}
$$

where $\sum_{i=1}^{n} \delta_{i}=1$.

Proof: Expanding $\left(\varrho_{i}-\varrho_{j}\right)^{T} P\left(\varrho_{i}-\varrho_{j}\right) \geq 0(i, j=$ $1,2, \cdots, n)$ gives

$$
\varrho_{i}^{T} P \varrho_{j}+\varrho_{j}^{T} P \varrho_{i} \leq \varrho_{i}^{T} P \varrho_{i}+\varrho_{j}^{T} P \varrho_{j},
$$

then, for any scalars $\delta_{i} \geq 0$ subject to $\sum_{i=1}^{n} \delta_{i}=1$ and any $\varrho_{i}$, one has

$$
\begin{aligned}
& \left(\sum_{i=1}^{n} \delta_{i} \varrho_{i}\right)^{T} P\left(\sum_{i=1}^{n} \delta_{i} \varrho_{i}\right) \\
& =\sum_{i=1}^{n} \delta_{i}^{2} \varrho_{i}^{T} P \varrho_{i}+\sum_{i=1}^{n} \sum_{j=1, j \neq i}^{n} \delta_{i} \delta_{j}\left(\varrho_{i}^{T} P \varrho_{j}+\varrho_{j}^{T} P \varrho_{i}\right) \\
& \leq \sum_{i=1}^{n} \delta_{i}^{2} \varrho_{i}^{T} P \varrho_{i}+\sum_{i=1}^{n} \sum_{j=1, j \neq i}^{n} \delta_{i} \delta_{j}\left(\varrho_{i}^{T} P \varrho_{i}+\varrho_{j}^{T} P \varrho_{j}\right) \\
& =\left(\delta_{1}+\ldots+\delta_{n}\right) \sum_{i=1}^{n} \delta_{i} \varrho_{i}^{T} P \varrho_{i} \\
& =\sum_{i=1}^{n} \delta_{i} \varrho_{i}^{T} P \varrho_{i} .
\end{aligned}
$$

Then, the proof is completed.

Lemma 2: Let the matrix $C \in \mathbb{R}^{m \times n}$ be of full-row rank. There always exist two orthogonal matrices $U \in \mathbb{R}^{m \times m}$ and $V \in \mathbb{R}^{n \times n}$ such that

$$
\left\{\begin{array}{l}
\tilde{C}:=U C V=U C\left[\begin{array}{ll}
V_{1} & V_{2}
\end{array}\right]=\left[\begin{array}{ll}
\Sigma & 0
\end{array}\right], \\
\Sigma=\operatorname{diag}\left\{\sigma_{1}, \sigma_{2}, \ldots, \sigma_{m}\right\}
\end{array}\right.
$$


where $V_{1} \in \mathbb{R}^{n \times m}$ and $V_{2} \in \mathbb{R}^{n \times(n-m)}, \sigma_{i}(i=1,2, \ldots, m)$ are nonzero singular values of $C$. Thus, if matrix $W$ has the following structure

$$
\begin{aligned}
W & =\left[\begin{array}{ll}
V_{1} & V_{2}
\end{array}\right]\left[\begin{array}{cc}
W_{1} & 0 \\
0 & W_{2}
\end{array}\right]\left[\begin{array}{l}
V_{1}^{T} \\
V_{2}^{T}
\end{array}\right] \\
& =V_{1} W_{1} V_{1}^{T}+V_{2} W_{2} V_{2}^{T}
\end{aligned}
$$

where $W_{1} \in \mathbb{R}^{m \times m}>0, W_{2} \in \mathbb{R}^{(n-m) \times(n-m)}>0$, then there exists a nonsingular matrix $\tilde{W} \in \mathbb{R}^{m \times m}$ such that $\tilde{W} C=C W$.

Proof: The proof is similar to that of Lemma 2 in [23], and is omitted here.

Note that Lemma 2 plays a critical role to handle the equality condition in the subsequent stability analysis.

\section{Main Results}

In this section, for all admissible parameter uncertainties and packet dropouts, the stability of the polytopic uncertain discrete-time linear system without constraints is firstly discussed and the desired SOF MPC controller is obtained by solving an optimization problem. Then, in terms of established results, the stability analysis and the controller design for the case with constraints are addressed by changing the corresponding constraints into linear matrix inequality (LMI) conditions.

\section{A. Unconstrained SOF MPC}

The following lemma will be used in deriving our main results.

Lemma 3: Let the symmetric positive-definite matrices $Q$ and $R$ be given. For system (1) controlled by (3), if there exist a positive scalar $\gamma(k)$, two symmetric positive definite matrices $W(k)$ and $\tilde{W}(k)$, and a matrix $Y(k)$ such that, for $l=1,2, \ldots, L$, the LMI (10) (at the bottom of this page) and

$$
\tilde{W}(k)\left[\begin{array}{lll}
C_{1}^{(l)} & \ldots & C_{N}^{(l)}
\end{array}\right]=\left[\begin{array}{lll}
C_{1}^{(l)} & \ldots & C_{N}^{(l)}
\end{array}\right] \bar{W}(k)
$$

hold, then we have

$$
\begin{aligned}
& \mathbb{E}[V(k+n+1)]-V(k+n) \\
< & -x^{T}(k+n \mid k) Q x(k+n \mid k)-u^{T}(k+n \mid k) R u(k+n \mid k)
\end{aligned}
$$

where

$$
\begin{aligned}
& V(k+n)=x^{T}(k+n \mid k) P(k) x(k+n \mid k), \quad n \geq 0 \\
& \tilde{\Gamma}^{(l)}=\tilde{\alpha}_{1} C_{1}^{(l)}+\sum_{i=2}^{N}\left[\prod_{j=1}^{i-1}\left(1-\tilde{\alpha}_{j}\right) \tilde{\alpha}_{i} C_{i}^{(l)}\right] \\
& \bar{W}(k)=\operatorname{diag}\{\underbrace{W(k), \ldots, W(k)}_{N}\}, P(k)=\gamma(k) W^{-1}(k) .
\end{aligned}
$$

Proof: See Appendix A.

Remark 2: The inequality condition (12), which provides a connection between the stability analysis and the cost function, has been widely applied in MPC issues. In this paper, such a condition is dependent of the redundant channels as well as its statistical characteristic.

It is obvious that if condition (12) holds, then $\lim _{n \rightarrow \infty} x(k+$ $n \mid k)=0$, and hence $V(\infty)=0$. Summing up both sides of (12) from $n=0$ to $n=\infty$ yields

$$
J(k) \leq V(k \mid k)=x^{T}(k) P(k) x(k)
$$

which leads to the following optimization problem:

$$
\text { OP1 : } \quad \max _{A^{(l)}, B^{(l)}, C_{i}^{(l)}(i=1,2, \ldots, N) \in \Omega} J(k) \leq V(k \mid k)
$$

The OP1 problem provides an upper bound on the ro-

\begin{tabular}{|c|c|c|c|c|c|c|c|c|c|c|}
\hline$W(k)$ & $*$ & $*$ & * & * & $*$ & $*$ & $*$ & $*$ & * & $*$ \\
\hline$\sqrt{Q} W(k)$ & $\gamma(k) I$ & * & $*$ & * & * & $*$ & * & * & * & * \\
\hline$A^{(l)} W(k)+B^{(l)} Y(k) \tilde{\Gamma}^{(l)}$ & 0 & $W(k)$ & $*$ & $*$ & * & $*$ & * & * & * & * \\
\hline$\sqrt{\tilde{\alpha}_{1}} B^{(l)} Y(k) C_{1}^{(l)}$ & 0 & 0 & $W(k)$ & $*$ & $*$ & $*$ & $*$ & $*$ & $*$ & $*$ \\
\hline$\sqrt{\tilde{\alpha}_{1} R} Y(k) C_{1}^{(l)}$ & 0 & 0 & 0 & $\gamma(k) I$ & $*$ & $*$ & $*$ & $*$ & $*$ & $*$ \\
\hline$\sqrt{\left(1-\tilde{\alpha}_{1}\right) \tilde{\alpha}_{2}} B^{(l)} Y(k) C_{2}^{(l)}$ & 0 & 0 & 0 & 0 & $W(k)$ & $*$ & $*$ & $*$ & $*$ & $*$ \\
\hline$\vdots$ & $\vdots$ & $\vdots$ & $\vdots$ & $\vdots$ & $\vdots$ & $\cdot$ & * & $*$ & * & $*$ \\
\hline$\prod_{j=1}^{N-1} \sqrt{\left(1-\tilde{\alpha}_{j}\right) \tilde{\alpha}_{N}} B^{(l)} Y(k) C_{N}^{(l)}$ & 0 & 0 & 0 & 0 & 0 & 0 & $W(k)$ & $*$ & $*$ & * \\
\hline$\sqrt{\left(1-\tilde{\alpha}_{1}\right) \tilde{\alpha}_{2} R} Y(k) C_{2}^{(l)}$ & 0 & 0 & 0 & 0 & 0 & 0 & 0 & $\gamma(k) I$ & $*$ & $*$ \\
\hline & $\vdots$ & $\vdots$ & $\vdots$ & $\vdots$ & $\vdots$ & : & $\vdots$ & $\vdots$ & $\ddots$ & $*$ \\
\hline$\prod_{j=1}^{N-1} \sqrt{\left(1-\tilde{\alpha}_{j}\right) \tilde{\alpha}_{N} R} Y(k) C_{N}^{(l)}$ & 0 & 0 & 0 & 0 & 0 & 0 & 0 & 0 & 0 & $\gamma(k) I$ \\
\hline
\end{tabular}
bust performance objective. Furthermore, noting that $P(k)=$ $\gamma(k) W^{-1}(k)$, one has $V(k) \leq \gamma(k)$ if

$$
\left[\begin{array}{cc}
1 & x^{T}(k) \\
x(k) & W(k)
\end{array}\right] \geq 0 .
$$

It is clear from (12) and (15) that $\Pi(P(k), \gamma(k))=\{x(k) \in$ $\left.\mathbb{R}^{n_{x}}: \mathbb{E}\left\{x^{T}(k) W^{-1}(k) x(k)\right\} \leq \gamma(k)\right\}$ is an invariant ellipsoid in mean-square sense for the predicted states of the uncertain system. Furthermore, the unconstrained SOF MPC controller for the polytopic uncertain discrete-time linear 
system with $N$ redundant channels is designed by proceeding with the following minimization problem in terms of some LMIs at each instant $k$ :

OP2 : $\min _{W(k), \tilde{W}(k)>0, Y(k)} \gamma(k)$, s.t. $(10),(11)$ and $(15)$.

It should be noted that once the minimization is solved, only the first prediction input $u(k)$ will be implemented.

Now, we are ready to give the main result for the closedloop system without constraints.

Theorem 1: Let the symmetric positive-definite matrices $Q$ and $R$ be given. Consider the unconstrained discrete-time linear system with N-redundant-channel described by (1)-(3). For the initial state $x(k)$ at time instant $k$, if there is a feasible solution to the optimization problem (OP2), then there also exists a feasible solution at time instant $t \geq k$. Furthermore, the closed-loop system with the unconstrained SOF MPC control law $F(k)=Y(k) \tilde{W}^{-1}(k)$ is locally stable in meansquare sense and the cost function $J(k)$ has an upper bound $\gamma(k)$ at time $k$.

Proof: Along the similar line in [13], the proof on stability can be divided into three steps. The first step is to show that an ellipsoid $\Pi(P(k), \gamma(k))$ is an invariant set in mean-square sense for the predicted state $x(k+n \mid k)$, where $P(k)$ and $\gamma(k))$ is the solution of the optimization problem (OP2). The second step is to discuss the feasibility of (OP2) for any $t>k$ when it is solvable at time $k$, and the last step is to prove the stability in mean-square sense in light of the invariant set and the feasibility of (OP2). Let us now go ahead step by step.

1) Invariant set: According to (12) in Lemma 3 and (15), one has

$$
\begin{aligned}
& \mathbb{E}\left\{x^{T}(k+n+1 \mid k) P(k) x(k+n+1 \mid k)\right\} \\
< & \mathbb{E}\left\{x^{T}(k+n \mid k) P(k) x(k+n \mid k)\right\}<\gamma(k)
\end{aligned}
$$

which means that $\Pi(P(k), \gamma(k))$ is an invariant set for the predicted state $x(k+n \mid k)$.

2) Feasibility: Assuming that the optimization problem (OP2) is feasible at time $k$, we need only prove that such an optimization problem is also feasible for all future states $x(k+n \mid k+n)$. Firstly, one has the relation

$$
\begin{aligned}
& x(k+n \mid k+n)=x(k+n), \\
& x(k+1 \mid k)=(A(k)+B(k) F(k) \Gamma(k)) x(k \mid k) \\
& x(k+1)=(A(k)+B(k) F(k) \Gamma(k)) x(k), \\
& \quad \text { for some }(A(k), B(k)) \in \Omega
\end{aligned}
$$

Then, noting the invariant set $\Pi(P(k), \gamma(k))$, the solution of the optimization problem (OP2) at time $k$ satisfies

$$
\mathbb{E}\left\{x^{T}(k+n \mid k) P(k) x(k+n \mid k)\right\}<\gamma(k), n \geq 1
$$

which, by using (16), implies

$$
\mathbb{E}\left\{x^{T}(k+1 \mid k+1) P(k) x(k+1 \mid k+1)\right\}<\gamma(k) .
$$

Furthermore, it is obvious that only the constraint (15) in problem (OP2) is dependent of the states, and thus LMIs (10), (11) and (15) are true at time $k+1$. In other words, the feasible solution at time $k$ is also feasible at time $k+1$. This process can be continued for all time.
3) Stability: In order to prove the stability, we only need to show that the quadratic function $\tilde{V}(x(k \mid k))=$ $x^{T}(k \mid k) P^{*}(k) x(k \mid k)$ is a strictly decreasing in mean-square sense, where $P^{*}(k)$ is the optimal solution of problem (OP2). Firstly, according to the part of Feasibility, one has

$$
\begin{aligned}
& \mathbb{E}\left\{x^{T}(k+1 \mid k+1) P^{*}(k+1) x(k+1 \mid k+1)\right\} \\
< & \mathbb{E}\left\{x^{T}(k+1 \mid k+1) P^{*}(k) x(k+1 \mid k+1)\right\}
\end{aligned}
$$

On the other hand, we know from the part of Invariant set that if $u(k+n \mid k)=F(k) y(k+n \mid k)(F(k)$ is obtained from the optimal solution at time $k$, one has

$$
\begin{aligned}
& \mathbb{E}\left\{x^{T}(k+1 \mid k) P^{*}(k) x(k+1 \mid k)\right\} \\
< & \mathbb{E}\left\{x^{T}(k \mid k) P^{*}(k) x(k \mid k)\right\}, \quad x(k \mid k) \neq 0
\end{aligned}
$$

Noting (16), and combining inequalities (17) and (18), we have

$$
\begin{aligned}
& \mathbb{E}\left\{x^{T}(k+1 \mid k+1) P^{*}(k+1) x(k+1 \mid k+1)\right\} \\
< & \mathbb{E}\left\{x^{T}(k \mid k) P^{*}(k) x(k \mid k)\right\}
\end{aligned}
$$

Therefore, $x^{T}(k \mid k) P^{*}(k) x(k \mid k)$ is strictly decreasing in mean-square sense for the closed-loop system. This means that $x(k) \rightarrow 0$ as $k \rightarrow \infty$. Finally, according to Lemma 3 , it is not difficult to see that the cost function $J(k)$ has an upper bound $\gamma(k)$ at time $k$, which completes the proof.

Remark 3: In this paper, we have examined how the input and output constraints, polytopic uncertainties and redundant channel transmission protocols influence the performance of the controlled systems in terms of MPC scheme. In Theorem 3 , all the system parameters as well as the occurrence probabilities for channels and the vertices of polyhedral domains are reflected in the linear matrix inequalities and therefore the proposed iterative algorithm shows robustness and reliability features. In the following section, an example is provided to show the usefulness and reliability of the proposed control scheme.

\section{B. Constrained SOF MPC}

At first, we give two transformations for input and output constraints (4a)-(4b). And then, by incorporating these transformed constraints into the optimization (OP2), the stability criterion for polytopic uncertain discrete-time linear system with both control input and measurement output constraints will be presented.

Lemma 4: The constraints on the control input and the measurement output (4a) and (4b) are satisfied if there exist three symmetric and positive definite matrices $W(k), \mathcal{X}(k)$, $\mathcal{Y}(k)$ and one matrix $Y(k)$ such that, for $p=1,2, \ldots, n_{u}$ and $q=1,2, \ldots, n_{y}$, the following inequalities:

$$
\left\{\begin{array}{c}
{\left[\begin{array}{cc}
\mathcal{X}(k) & Y(k) \Upsilon^{(l)} \\
* & W(k)
\end{array}\right] \geq 0, \quad[\mathcal{X}(k)]_{p p} \leq \bar{u}_{p}^{2}} \\
{\left[\begin{array}{cc}
\mathcal{Y}(k) & \Delta^{(l)} \\
* & W(k)
\end{array}\right] \geq 0, \quad[\mathcal{Y}(k)]_{q q} \leq \bar{y}_{q}^{2}}
\end{array}\right.
$$


hold, where $[\mathcal{X}(k)]_{p p}\left([\mathcal{Y}(k)]_{q q}\right)$ is the $p$ th $(q$ th $)$ diagonal element of matrix $\mathcal{X}(k)(\mathcal{Y}(k))$ and

$$
\begin{aligned}
& \Upsilon^{(l)}=\alpha_{1}(k) C_{1}^{(l)}+\sum_{i=2}^{N}\left\{\prod_{j=1}^{i-1}\left(1-\alpha_{j}(k)\right) \alpha_{i}(k) C_{i}^{(l)}\right\} \\
& \Theta_{i}^{(l)}=A^{(l)} W(k)+B^{(l)} Y(k) C_{i}^{(l)}, i=1,2, \ldots, N \\
& \Delta^{(l)}=\alpha_{1}(k) C_{1}^{(l)} \Theta_{1}^{(l)}+\sum_{i=2}^{N}\left\{\prod_{j=1}^{i-1}\left(1-\alpha_{j}(k)\right) \alpha_{i}(k) C_{i}^{(l)} \Theta_{i}^{(l)}\right\} .
\end{aligned}
$$

Proof: For the control input constraint (4a), it follows from (11) and Lemma 1 that

$$
\begin{aligned}
& \left|[u(k+n \mid k)]_{p}\right|^{2} \\
= & \left|[F(k) y(k+n \mid k)]_{p}\right|^{2} \\
= & \mid e_{p} F(k)\left\{\alpha_{1}(k) C_{1}(k+n)+\sum_{i=2}^{N}\left(\prod_{j=1}^{i-1}(1\right.\right. \\
& \left.\left.\left.-\alpha_{j}(k)\right) \alpha_{i}(k) C_{i}(k+n)\right)\right\}\left.x(k+n \mid k)\right|^{2} \\
\leq & \sum_{l=1}^{L} \delta_{l}\left|e_{p} Y(k) \tilde{W}^{-1}(k) \Upsilon^{(l)} x(k+n \mid k)\right|^{2} \\
\leq & \sum_{l=1}^{L} \delta_{l}\left|e_{p} Y(k) \Upsilon^{(l)} W^{-1 / 2}(k) W^{-1 / 2}(k) x(k+n \mid k)\right|^{2}
\end{aligned}
$$

where $e_{p}$ is the $p$ th row of an $n_{u}$-ordered identity matrix. Furthermore, by utilizing the Cauchy-Schwarz inequality and (15), one has

$$
\begin{aligned}
& \left|[u(k+n \mid k)]_{p}\right|^{2} \\
\leq & \sum_{l=1}^{L} \delta_{l}\left\|e_{p} Y(k) \Upsilon^{(l)} W^{-1 / 2}(k)\right\|^{2} \\
& \times\left\|W^{-1 / 2}(k) x(k+n \mid k)\right\|^{2} \\
\leq & \sum_{l=1}^{L} \delta_{l}\left\|e_{p} Y(k) \Upsilon^{(l)} W^{-1 / 2}(k)\right\|^{2} \\
= & \sum_{l=1}^{L} \delta_{l} e_{p} Y(k) \Upsilon^{(l)} W^{-1}(k)\left(Y(k) \Upsilon^{(l)}\right)^{T} e_{p}^{T}
\end{aligned}
$$

By using the Schur Complement Lemma, one can obtain $\left|[u(k+n \mid k)]_{p}\right| \leq \bar{u}_{p}$ when the inequality (20a) is true.

In what follows, by applying the same method, we can obtain that the constraint on measurement output described by (4b) satisfies

$$
\begin{aligned}
& \left|[y(k+n \mid k)]_{q}\right|^{2} \\
\leq & \sum_{l=1}^{L} \delta_{l} \mid e_{q}\left\{\alpha_{1}(k) C_{1}^{(l)}\left(A^{(l)}+B^{(l)} F(k) C_{1}^{(l)}\right)\right. \\
& +\sum_{i=2}^{N}\left(\prod _ { j = 1 } ^ { i - 1 } ( 1 - \alpha _ { j } ( k ) ) \alpha _ { i } ( k ) C _ { i } ^ { ( l ) } \left(A^{(l)}\right.\right. \\
& \left.\left.\left.+B^{(l)} F(k) C_{i}^{(l)}\right)\right)\right\}\left.x(k+n \mid k)\right|^{2} \\
= & \sum_{l=1}^{L} \delta_{l}\left|e_{q} \Delta^{(l)} W^{-1 / 2}(k) W^{-1 / 2}(k) x(k+n \mid k)\right|^{2} \\
\leq & \sum_{l=1}^{L} \delta_{l}\left\|e_{q} \Delta^{(l)} W^{-1 / 2}(k)\right\|^{2} \\
= & \sum_{l=1}^{L} \delta_{l} e_{q} \Delta^{(l)} W^{-1}(k) \Delta^{(l)^{T}} e_{q}^{T} .
\end{aligned}
$$

By using the Schur complement Lemma again, one can obtain $\left|[y(k+n \mid k)]_{q}\right| \leq \bar{y}_{q}$ when the inequality (20b) is true, which completes the proof.

After the transformation of the constraints into LMIs, the corresponding optimization problem on SOF MPC subject to both control and output constraints can be presented as follows

$$
\text { OP3 : } \min _{\{W(k), \tilde{W}(k), \mathcal{X}(k), \mathcal{Y}(k)\}>0, Y(k)} \gamma(k),
$$

$$
\text { s.t. }(10),(11),(15),(20 a) \text { and }(20 b) \text {. }
$$

For addressed problem with both input and output constraints, we have the following theorem whose proof is similar to Theorem 1, and thus is omitted here.

Theorem 2: Let the symmetric positive-definite matrices $Q$ and $R$ be given. Consider the uncertain discrete-time linear system with N-redundant-channel, and both input and output constraints described by (1)-(4b). For the initial state $x(k)$ at time instant $k$, if there is a feasible solution to the optimization problem (OP3), there also exists a feasible solution at time instant $t \geq k$ and the closed-loop system with the constrained SOF MPC control law $F(k)=Y(k) \tilde{W}^{-1}(k)$ is locally stable in mean-square sense. Furthermore, the cost function $J(k)$ has an upper bound $\gamma(k)$ at time $k$.

It is noted that, for the output-feedback control problem considered in this paper, the state $x(k)$ is not guaranteed to be measurable and the condition (15) cannot be conveniently verified in practice. To deal with this issue, we provide some easy-to-test conditions ensuring the validity of (15) in the following lemma.

Lemma 5: Consider system (5). If there are positive definite matrices $W(k), M(k)$ and matrices $Y(k)$ such that the following inequalities

$$
\begin{gathered}
{\left[\begin{array}{cc}
W(k) & * \\
A^{(l)} W(k)+B^{(l)} Y(k) \Upsilon(l) & M(k)
\end{array}\right] \geq 0,} \\
W(k) \geq M(k), W(k) \geq S, x_{0}^{T} S^{-1} x_{0} \leq 1,
\end{gathered}
$$


hold where $x_{0}$ is the initial state and matrix $S>0$ is given according to the engineering requirement, then the condition (15) is true.

Proof: Let us assume that the conditions (24) and (25) are satisfied at $k=0$. It is easy to see that $\left\|x_{0}\right\|_{W-1}^{2} \leq$ $\left\|x_{0}\right\|_{M^{-1}}^{2} \leq\left\|x_{0}\right\|_{S^{-1}}^{2} \leq 1$, which implies that the condition (15) is satisfied at time $k=0$. Now, for the case of $k=1$, according to (5) and (24), we can obtain that $\|x(1 \mid 0)\|_{M^{-1}}^{2} \leq$ $\left\|x_{0}\right\|_{W^{-1}}^{2}$. Furthermore, considering condition (25) as well as the conclusion at $k=0$, we have $\|x(1 \mid 0)\|_{W^{-1}}^{2} \leq\left\|x_{0}\right\|_{S^{-1}}^{2} \leq$ 1. Based on the definition of invariant set in the proof of Theorem 1, it is concluded that $\|x(1)\|_{W^{-1}}^{2} \leq 1$, which means that the condition (15) is also true at $k=1$. Such a procedure can be repeated and we can eventually prove that (15) is true at all times.

Due to the equality constraint (11), it would not be easy to solve the optimization problem (OP2) or (OP3). In order to overcome such a difficulty, the following theorem is proposed with the aid of Lemma 2.

Theorem 3: Let the symmetric positive-definite matrices $Q$ and $R$ be given and the matrices $C_{1}^{(l)}=C_{2}^{(l)}=\ldots=$ $C_{N}^{(l)}=C(l=1,2, \cdots, L)$ be of full-row rank. Consider the uncertain discrete-time linear system with N-redundantchannel as well as the input and output constraints described by (1)-(4b). For the initial state $x(k)$ at time instant $k$, assume that there exist positive scalar $\gamma(k)$, symmetric matrices $\mathcal{X}(k), \mathcal{Y}(k), W_{1}(k), W_{2}(k)$ and any matrix $Y(k)$ such that the following optimization problem at time instant $k$ is feasible for the initial state $x(k)$ :

$$
\begin{aligned}
& \text { OP4 : } \min _{\left\{W_{1}(k), W_{2}(k), M(k), \mathcal{X}(k), \mathcal{Y}(k)\right\}>0, Y(k)} \gamma(k) \text {, } \\
& \text { s.t. }(10),(20 a),(20 b),(24) \text { and }(25) \text {. }
\end{aligned}
$$

Then, there also exists a feasible solution at time instant $t \geq k$. Furthermore, the closed-loop system with the constrained SOF MPC control law

$$
F(k)=Y(k) U^{T} \Sigma W_{1}^{-1}(k) \Sigma^{-1} U
$$

is locally stable in mean-square sense and the cost function $J(k)$ has an upper bound $\gamma(k)$ at time $k$ where $W(k)=$ $V_{1} W_{1}(k) V_{1}^{T}+V_{2} W_{2}(k) V_{2}^{T}, U, \Sigma, V_{1}$ and $V_{2}$ come from the singular value decomposition of matrix $C$ satisfying $U C\left[\begin{array}{ll}V_{1} & V_{2}\end{array}\right]=\left[\begin{array}{cc}\Sigma & 0\end{array}\right]$.

Proof: Noting $W(k)=V_{1} W_{1}(k) V_{1}^{T}+V_{2} W_{2}(k) V_{2}^{T}$, one has from Lemma 2 that

$$
U^{T}\left[\begin{array}{ll}
\Sigma & 0
\end{array}\right] V^{T} W(k)=\tilde{W}(k) U^{T}\left[\begin{array}{ll}
\Sigma & 0
\end{array}\right] V^{T}
$$

that is

$U^{T}\left[\begin{array}{ll}\Sigma & 0\end{array}\right] V^{T} V\left[\begin{array}{cc}W_{1}(k) & 0 \\ 0 & W_{2}(k)\end{array}\right] V^{T}=\tilde{W}(k) U^{T}[\Sigma$

It is easily obtained that

$$
\tilde{W}^{-1}(k)=U^{T} \Sigma W_{1}^{-1}(k) \Sigma^{-1} U .
$$

Therefore, if the optimization problem (OP4) is solvable, the equality constraint (11) holds. The rest of the proof follows from Theorem 2 and the proof is now complete.
Remark 4: It is easy to see that the equality constraint (11) is now excluded in the optimization problem (OP4). Therefore, the optimization problem (OP4) can be effectively solved by using Matlab LMI toolbox, where the singular value decomposition approach plays a critical role in dealing with this optimization problem.

Remark 5: As we know, the algorithm based on the standard LMI system has a polynomial-time complexity. Specifically, the number $\mathcal{N}(\varepsilon)$ of flops needed to compute an $\varepsilon$ accurate solution is bounded by $O\left(\mathcal{M N}^{3} \log (\mathcal{V} / \varepsilon)\right)$, where $\mathcal{M}$ is the total row size of the LMIs of the proposed algorithm, $\mathcal{N}$ is the total number of scalar decision variables, $\mathcal{V}$ is a data-dependent scaling factor, and $\varepsilon$ is the relative accuracy set for algorithm [5]. For the discussed closedloop system (5), the variables dimensions can be seen from $x(k) \in \mathbb{R}^{n_{x}}, u(k) \in \mathbb{R}^{n_{u}}$ and $y(k) \in \mathbb{R}^{n_{y}}$. Furthermore, notice that the optimization problem (OP4) involves conditions (10), (20a), (20b), (24) and (25), one has $\mathcal{M}=$ $[(7+N) L+3] n_{x}+[(N+1) L+1] n_{y}+(L+1) n_{u}$ and $\mathcal{N}=\left(n_{x}+1\right) n_{x}+\frac{1}{2}\left(n_{u}+1\right) n_{u}+n_{u} n_{y}+1$. Thus, the computational complexity of the presented algorithm can be represented as $O\left(N L n_{x}^{7}\right)$. Obviously, such a computational complexity depends polynomially on the variable dimensions.

\section{Algorithm for constrained SOF MPC}

\begin{tabular}{|c|c|}
\hline \multicolumn{2}{|c|}{ Algorithm: } \\
\hline \multicolumn{2}{|c|}{ Off-line part } \\
\hline Step 1. & $\begin{array}{l}\text { For the given matrices } C=C_{1}^{(l)}=\ldots=C_{N}^{(l)}, l= \\
1,2, \ldots, L, \text { find two orthogonal matrices } U \text { and } V \text { to satisfy } \\
\text { the conditions in Lemma } 2 \text {. }\end{array}$ \\
\hline \multicolumn{2}{|c|}{ Online part } \\
\hline Step 2. & Choose initial state $x_{0}$ and proper matrix $S>0$. \\
\hline Step 3. & $\begin{array}{l}\text { At sampling instant } k \text {, measure the plant output and solve the } \\
\text { optimization problem (OP4). }\end{array}$ \\
\hline Step 4. & $\begin{array}{l}\text { For the latest measure outputs, calculate } u(k) \text { by (3) and (26), } \\
\text { and then act it on the plant. Set the time } k=k+1 \text { and go } \\
\text { back to Step } 3 \text {. }\end{array}$ \\
\hline
\end{tabular}

To present the algorithm for constrained SOF MPC, we assume that the matrices $C=C_{1}^{(l)}=\ldots=C_{N}^{(l)}, l=1,2, \ldots, L$ with full-row rank. The proposed algorithm includes two parts, that is, Off-line part and Online part.

Remark 6: If the optimization (OP4) is feasible at time $k$, then the first computed input $u(k)=F(k) y(k \mid k)$ is implemented on the controlled plant. At the next instant, the output $y(k+1)$ is measured and the optimization is repeated to compute $F(k+1)$.

Remark 7: In this paper, for the purpose of the discussion convenience and the model simplification, the sampling period is designed according to the online optimization time of MPC algorithm. In other words, the online optimization time, the bounded of which can be determined in advance, is less than sampling period. Such a method avoids the waiting step of sampling period duration. Furthermore, in order to obtain the desired controller gain, Lemma 2, which is essentially a singular decomposition, is developed. In comparison with ones 


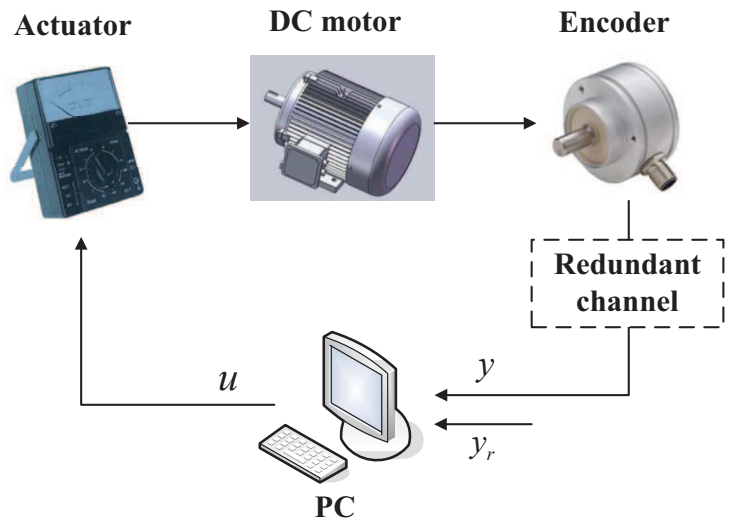

Fig. 2. System configuration for a DC motor with redundant channel transmission.

in [23], our condition in Lemma 2 is not a single equality constraint on matrix $C$, but a series of equality constraints on matrices $C_{i}$ due to the redundant channel framework application.

\section{Application to The DC Motor CONTROL SYSTEM}

In this section, a DC motor system is utilized to demonstrate the effectiveness of the proposed algorithm.

The loop equation for the electrical circuit and the mechanical torque balance in a DC motor system can be, respectively, described by [3]

$$
\begin{gathered}
\mathrm{u}(t)=e_{a}(t)=L \frac{\mathrm{d} i_{a}(t)}{\mathrm{d} t}+R i_{a}(t)+e_{b}(t), \\
J \frac{\mathrm{d} \omega(t)}{\mathrm{d} t}+B \omega(t)+T_{l}(t)=K i_{a}(t)
\end{gathered}
$$

where $\mathrm{u}(t)=e_{a}(t)$ is the armature winding input voltage; $e_{b}(t)=K_{b} \omega(t)$ is the back-electromotive-force (EMF) voltage; $L$ is the armature wingding inductance; $i_{a}(t)$ is the armature winding current; $R$ is the armature winding resistance; $B$ is the system damping coefficient; $K$ and $K_{b}$ are, respectively, the torque constant and the back-EMF constant; $T_{l}(t)$ is the load torque; and $\omega(t)$ is the rotor angular speed.

By letting $x_{1}(t)=i_{a}(t), x_{2}(t)=\omega(t), u_{1}(t)=e_{a}(t)$, $u_{2}(t)=T_{l}(t)$, the electromechanical dynamics of the DC motor (29)-(30) can be described by the following state-space description:

$$
\left\{\begin{array}{l}
\frac{\mathrm{d} x_{1}(t)}{\mathrm{d} t}=-\frac{R}{L} x_{1}(t)-\frac{K_{b}}{L} x_{2}(t)+\frac{1}{L} u_{1}(t) \\
\frac{\mathrm{d} x_{2}(t)}{\mathrm{d} t}=\frac{K}{J} x_{1}(t)-\frac{B}{J} x_{2}(t)-\frac{1}{J} u_{2}(t)
\end{array}\right.
$$

For the addressed example, the parameters of DC motor system are presented in the following Table I [3]:

By setting sampling period $T=0.05 \mathrm{~s}$ and denoting $x(t)=\left[\begin{array}{ll}x_{1}(t) & x_{2}(t)\end{array}\right]^{T}, u(t)=\left[\begin{array}{ll}u_{1}(t) & u_{2}(t)\end{array}\right]^{T}$ and $y(t)=\left[\begin{array}{ll}i_{a}(t) & \omega(t)\end{array}\right]^{T}=\left[\begin{array}{ll}x_{1}(t) & x_{2}(t)\end{array}\right]^{T}$, then the
TABLE I

DC MOTOR PARAMETERS

\begin{tabular}{ccc}
\hline Parameters & & Value \\
\hline$J$ & Inertia & $42.6 \times 10^{-6} \mathrm{~kg} \cdot \mathrm{m}^{2}$ \\
$L$ & Inductance & $0.170 \mathrm{H}$ \\
$R$ & Resistance & $4.67 \Omega$ \\
$B$ & Damping coefficient & $47.3 \times 10^{-6} \mathrm{~N} \cdot \mathrm{m} \cdot \mathrm{s} / \mathrm{rad}$ \\
$K$ & Torque constant & $14.7 \times 10^{-3} \mathrm{~N} \cdot \mathrm{m} / \mathrm{A}$ \\
$K_{e}$ & Back-EMF constant & $14.7 \times 10^{-3} \mathrm{~V} \cdot \mathrm{s} / \mathrm{rad}$ \\
\hline
\end{tabular}

system (31) can be discretized as follows:

$$
\left\{\begin{aligned}
x(k+1)= & {\left[\begin{array}{cc}
0.2379 & -0.0022 \\
8.96 & 0.9223
\end{array}\right] x(k) } \\
& +\left[\begin{array}{cc}
0.158 & 1.643 \\
1.643 & -1131
\end{array}\right] u(k) \\
\triangleq & A x(k)+B u(k) \\
y(k)= & {\left[\begin{array}{ll}
1 & 0 \\
0 & 1
\end{array}\right] x(k) \triangleq C x(k) }
\end{aligned}\right.
$$

For real-world systems, due to measurement inaccuracy and external disturbance, the parameter uncertainties are inevitable in system modeling. Thus, we assume that the system matrix $A$ of DC motor system is polytopic uncertain and the vertexes of polyhedral domain are

$$
\begin{gathered}
A^{(1)}=\left[\begin{array}{cc}
0.2379 & -0.0022 \\
8.96 & 0.9223
\end{array}\right], A^{(2)}=\left[\begin{array}{cc}
0.2879 & -0.0012 \\
9.23 & 0.8125
\end{array}\right], \\
B^{(1)}=\left[\begin{array}{cc}
0.158 & 1.643 \\
1.643 & -1131
\end{array}\right], B^{(2)}=\left[\begin{array}{cc}
0.098 & 0.965 \\
1.213 & -1246
\end{array}\right] .
\end{gathered}
$$

Furthermore, the number of redundant channels is $N=3$ and the measurement matrices are $C_{1}=C_{2}=C_{3}=C$. The probabilities of successfully transmitted packets on three channels are taken as $\tilde{\alpha}_{1}=0.7, \tilde{\alpha}_{2}=0.8$ and $\tilde{\alpha}_{3}=0.9$.

In this example, let the initial state and the reference output be $x_{0}=\left[\begin{array}{ll}0.3 & 3\end{array}\right]^{T}$ and $y_{r}=\left[\begin{array}{ll}3 & 300\end{array}\right]^{T}$. The weighting matrices are selected as $Q=R=I_{2}$, the constraints on control input and measurement output are considered as $\bar{u}=\left[\begin{array}{ll}30 & 0.3\end{array}\right]^{T}$ and $\bar{y}=\left[\begin{array}{ll}5 & 400\end{array}\right]^{T}$. The simulation results are shown in Figs. 3-6.

Fig. 3 describes the phenomena of packet dropouts for three channels with the aforementioned probabilities. Fig. 4 presents the upper-bound of cost function in 3 communication cases, that is, the case with only channel 1 , the case with channels 1 and 2, and the case with all channels. From this figure, we can easily see that the upper-bound with all channels is the smallest and tends to a stable value at the fastest speed. While, the upper-bound with only channel 1 is the biggest and can not converge to a certain value. Furthermore, the output responses under different communication cases are depicted in Fig. 5, in which we can find that the system output with all channels can track the reference very well, oppositely the system output with only channel 1 works terribly. Fig. 6 depicts the control inputs, in which the curve with all channels is the best. In a word, the more channels are employed, the better performance will be obtained. This example validates the effectiveness of 


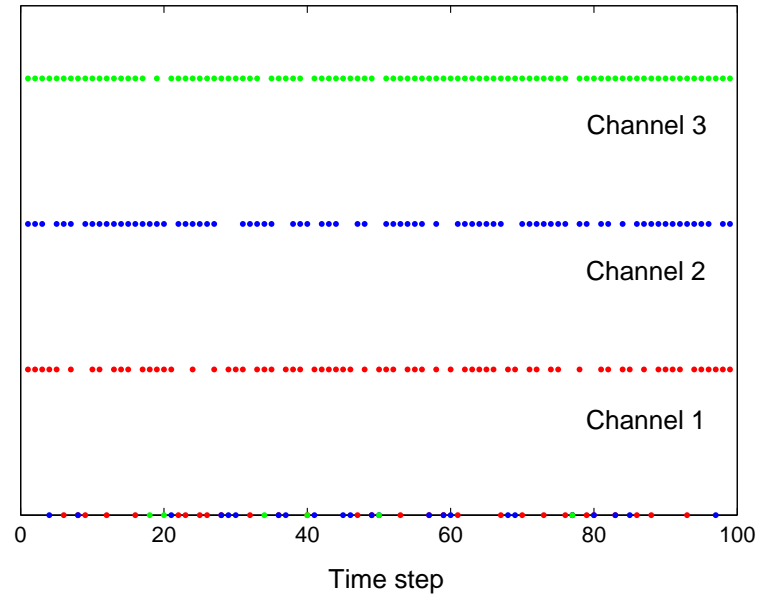

Fig. 3. Random packet dropouts in three channels.

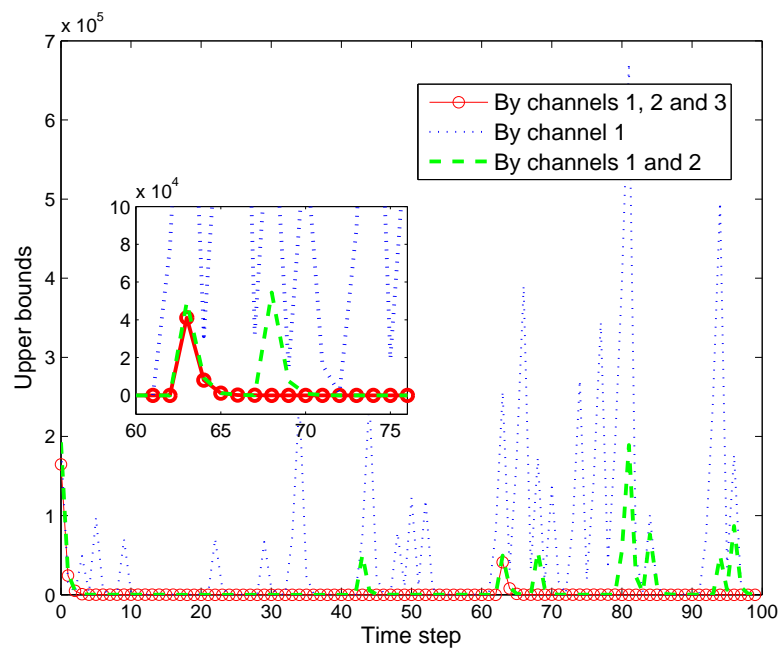

Fig. 4. Upper bounds under different communication cases.

the proposed SOF MPC algorithm in guaranteeing the system stability and in improving the system performance.

\section{CONCLUSION}

In this paper, the SOF MPC problem has been investigated for a class of polytopic uncertain discrete-time linear systems with redundant channels, and input and output constraints. With the aid of a set of Bernoulli distributed random variables, a new measurement model has been proposed to describe the redundant channel communication protocol. Based on such a model, some optimization problems have been presented step by step to carry out the feasibility analysis of designed MPC scheme and the robust stability analysis of the closedloop system. Furthermore, by using the singular value decomposition approach, an easy-to-use MPC algorithm has been provided to obtain the desired sub-optimal control sequence and an upper-bound of quadratic cost function. Finally, a DC motor system with redundant channels has been exploited to show the effectiveness of the SOF MPC scheme proposed

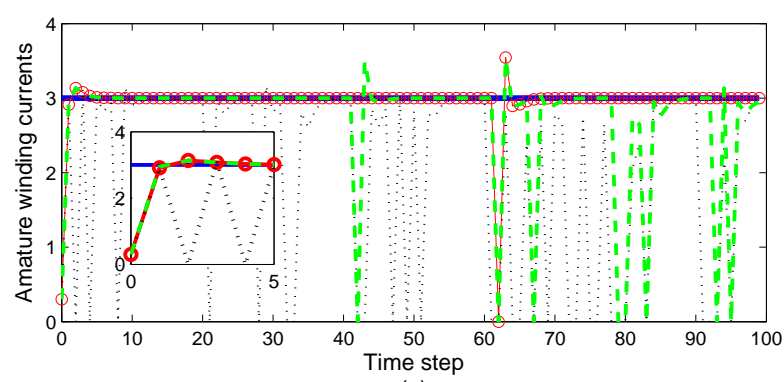

(a)

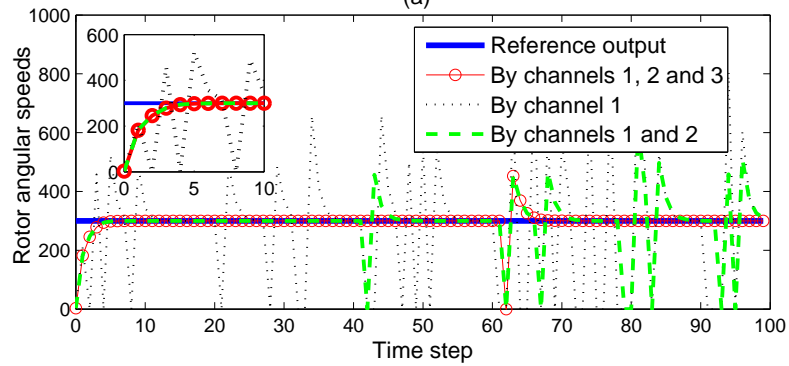

(b)

Fig. 5. Output responses under different communication cases.

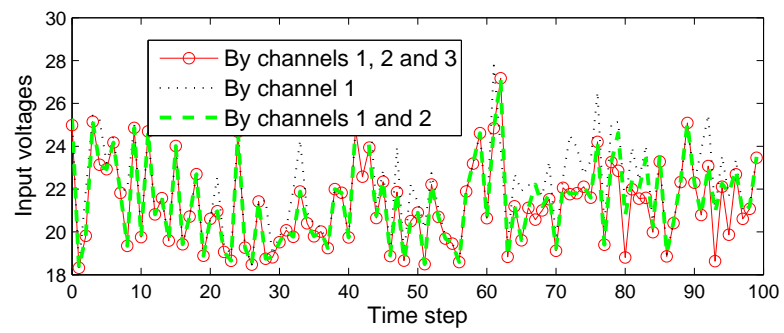

(a)

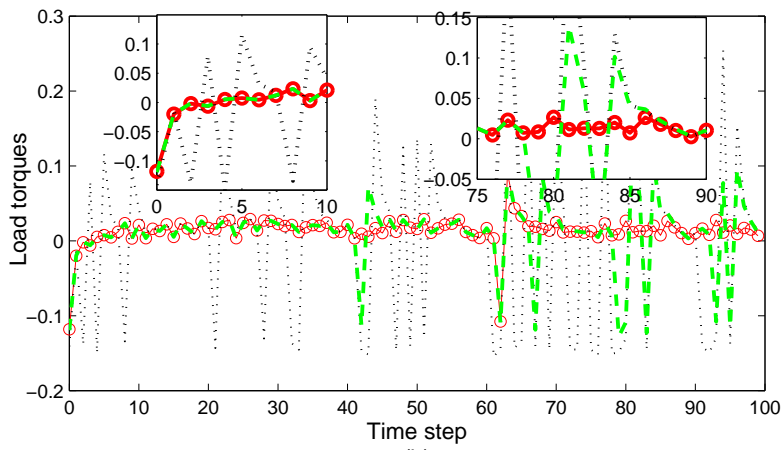

(b)

Fig. 6. Control inputs under different communication cases.

in this paper. In the future, we might further investigate the MPC problem with redundant-channel transmission subject to some other network-induced phenomena such as time-delay and quantization [6]-[9].

\section{APPENDIX}

\section{A: Proof of Lemma 3}

Consider the quadratic function as follows:

$V(k+n \mid k)=x^{T}(k+n \mid k) P(k) x(k+n \mid k), \quad n \geq 0$

Calculating the difference of $V(k+n \mid k)$ along the trajectory 
of (5) and taking the mathematical expectation, we have

$$
\begin{aligned}
& \mathbb{E}\{\Delta V(x(k+n \mid k))\} \\
&=\mathbb{E}\{V(k+n+1 \mid k)-V(k+n \mid k)\} \\
&=\mathbb{E}\left\{x^{T}(k+n+1 \mid k) P(k) x(k+n+1 \mid k)\right. \\
&\left.-x^{T}(k+n \mid k) P(k) x(k+n \mid k)\right\} \\
&= \mathbb{E}\left\{x ^ { T } ( k + n | k ) \left\{[A(k+n)+B(k+n) F(k) \Gamma(k+n)]^{T}\right.\right. \\
& \times P(k)[A(k+n)+B(k+n) F(k) \Gamma(k+n)] \\
&-P(k)\} x(k+n \mid k)\} \\
&= x^{T}(k+n \mid k)\left\{[A(k+n)+B(k+n) F(k) \bar{\Gamma}(k+n)]^{T}\right. \\
& \times P(k)[A(k+n)+B(k+n) F(k) \bar{\Gamma}(k+n)]-P(k) \\
&-[B(k+n) F(k) \bar{\Gamma}(k+n)]^{T} P(k) B(k+n) F(k) \\
& \times \bar{\Gamma}(k+n)+\tilde{\alpha}_{1}\left[B(k+n) F(k) C_{1}(k+n)\right]^{T} P(k) \\
& \times B(k+n) F(k) C_{1}(k+n)+\sum_{i=2}^{N}\left(\prod_{j=1}^{i-1}\left(1-\tilde{\alpha}_{j}\right) \tilde{\alpha}_{i}\right. \\
& \times\left[B(k+n) F(k) C_{i}(k+n)\right]^{T} P(k) \\
&\left.\left.\times B(k+n) F(k) C_{i}(k+n)\right)\right\} x(k+n \mid k) \\
& \leq x^{T}(k+n \mid k)\left\{[A(k+n)+B(k+n) F(k) \bar{\Gamma}(k+n)]^{T}\right. \\
& \times P(k)[A(k+n) B(k+n) F(k) \bar{\Gamma}(k+n)]-P(k) \\
&+\left[\sqrt{\tilde{\alpha}_{1}} B(k+n) F(k) C_{1}(k+n)\right]^{T} P(k) \\
& \times\left[\sqrt{\tilde{\alpha}_{1}} B(k+n) F(k) C_{1}(k+n)\right] \\
&+\sum_{i=2}^{N}\left(\prod_{j=1}^{i-1}\left(1-\tilde{\alpha}_{j}\right) \tilde{\alpha}_{i}\left(B(k+n) F(k) C_{i}(k+n)\right)^{T}\right. \\
&\left.\left.\times P(k) B(k+n) F(k) C_{i}(k+n)\right)\right\} x(k+n \mid k) \\
&(34)
\end{aligned}
$$

where

$\bar{\Gamma}(k+n)=\tilde{\alpha}_{1} C_{1}(k+n)+\sum_{i=2}^{N}\left[\prod_{j=1}^{i-1}\left(1-\tilde{\alpha}_{j}\right) \tilde{\alpha}_{i} C_{i}(k+n)\right]$.

In light of Lemma 1, the above inequality (34) holds if

$$
\begin{aligned}
& \mathbb{E}\{\Delta V(k+n \mid k)\} \\
\leq & \sum_{l=1}^{L} \delta_{l} x^{T}(k+n \mid k)\left\{( A ^ { ( l ) } + B ^ { ( l ) } F ( k ) \tilde { \Gamma } ^ { ( l ) } ) ^ { T } P ( k ) \left(A^{(l)}\right.\right. \\
& \left.+B^{(l)} F(k) \tilde{\Gamma}^{(l)}\right)-P(k)+\left(\sqrt{\tilde{\alpha}_{1}} B^{(l)} F(k) C_{1}^{(l)}\right)^{T} P(k) \\
& \times\left(\sqrt{\tilde{\alpha}_{1}} B^{(l)} F(k) C_{1}^{(l)}\right)+\sum_{i=2}^{N}\left(\prod_{j=1}^{i-1}\left(1-\tilde{\alpha}_{j}\right) \tilde{\alpha}_{i}\right. \\
& \left.\left.\times\left[B^{(l)} F(k) C_{i}^{(l)}\right]^{T} P(k) B^{(l)} F(k) C_{i}^{(l)}\right)\right\} x(k+n \mid k) .
\end{aligned}
$$

On the other hand, one can easily calculate

$$
\begin{aligned}
& -x^{T}(k+n \mid k) Q x(k+n \mid k)-u^{T}(k+n \mid k) R u(k+n \mid k) \\
= & \mathbb{E}\left\{-x^{T}(k+n \mid k) Q x(k+n \mid k)\right. \\
& \left.-[F(k) y(k+n \mid k)]^{T} R F(k) y(k+n \mid k)\right\}
\end{aligned}
$$

$$
\begin{aligned}
= & x^{T}(k+n \mid k)\left\{-Q-\left(\sqrt{\tilde{\alpha}_{1}} F(k) C_{1}(k+n)\right)^{T} R\right. \\
& \times\left(\sqrt{\tilde{\alpha}_{1}} F(k) C_{1}(k+n)\right)-\sum_{i=2}^{N}\left(\prod_{j=1}^{i-1}\left(1-\tilde{\alpha}_{j}\right) \tilde{\alpha}_{i}\right. \\
& \left.\left.\times\left(F(k) C_{i}(k+n)\right)^{T} R F(k) C_{i}(k+n)\right)\right\} x(k+n \mid k) \\
\leq & \sum_{l=1}^{L} \delta_{l} x^{T}(k+n \mid k)\left\{-Q-\left(\sqrt{\tilde{\alpha}_{1}} F(k) C_{1}^{(l)}\right)^{T} R\right. \\
& \times\left(\sqrt{\tilde{\alpha}_{1}} F(k) C_{1}^{(l)}\right)-\sum_{i=2}^{N}\left(\prod_{j=1}^{i-1}\left(1-\tilde{\alpha}_{j}\right) \tilde{\alpha}_{i}\left(F(k) C_{i}^{(l)}\right)^{T}\right. \\
& \left.\left.\times R F(k) C_{i}^{(l)}\right)\right\} x(k+n \mid k) .
\end{aligned}
$$

Taking (35) and (36) into consideration, one has

$$
\begin{aligned}
& \mathbb{E}[V(k+n+1 \mid k)]-V(k+n \mid k) \\
\leq & -x^{T}(k+n \mid k) Q x(k+n \mid k)-u^{T}(k+n \mid k) R u(k+n \mid k)
\end{aligned}
$$

when the following inequality is true

$$
\begin{aligned}
& x^{T}(k+n \mid k)\left\{[ A ^ { ( l ) } + B ^ { ( l ) } F ( k ) \tilde { \Gamma } ^ { ( l ) } ] ^ { T } P ( k ) \left[A^{(l)}\right.\right. \\
& \left.+B^{(l)} F(k) \tilde{\Gamma}^{(l)}\right]-P(k)+\left[\sqrt{\tilde{\alpha}_{1}} B^{(l)} F(k) C_{1}^{(l)}\right]^{T} P(k) \\
& \quad \times\left[\sqrt{\tilde{\alpha}_{1}} B^{(l)} F(k) C_{1}^{(l)}\right]+\left[\sqrt{\tilde{\alpha}_{1}} F(k) C_{1}^{(l)}\right]^{T} R\left[\sqrt{\tilde{\alpha}_{1}}\right. \\
& \left.F(k) C_{1}^{(l)}\right]+\sum_{i=2}^{N}\left(\prod_{j=1}^{i-1} \sqrt{\left(1-\tilde{\alpha}_{j}\right) \tilde{\alpha}_{i}}\left[B^{(l)} F(k) C_{i}^{(l)}\right]^{T}\right. \\
& \left.\quad \times P(k) \prod_{j=1}^{i-1} \sqrt{\left(1-\tilde{\alpha}_{j}\right) \tilde{\alpha}_{i}} B^{(l)} F(k) C_{i}^{(l)}\right) \\
& +\sum_{i=2}^{N}\left(\prod_{j=1}^{i-1} \sqrt{\left(1-\tilde{\alpha}_{j}\right) \tilde{\alpha}_{i}}\left[F(k) C_{i}^{(l)}\right]^{T} R\right. \\
& \left.\left.\quad \times \prod_{j=1}^{i-1} \sqrt{\left(1-\tilde{\alpha}_{j}\right) \tilde{\alpha}_{i}} F(k) C_{i}^{(l)}\right)+Q\right\} x(k+n \mid k) \\
& \leq 0
\end{aligned}
$$

In what follows, for nonzero $x(k+n \mid k)$, (37) is equivalent to

$$
\begin{aligned}
& {\left[A^{(l)}+B^{(l)} F(k) \tilde{\Gamma}^{(l)}\right]^{T} P(k)\left[A^{(l)}+B^{(l)} F(k) \tilde{\Gamma}^{(l)}\right]} \\
& +\left[\sqrt{\tilde{\alpha}_{1}} B^{(l)} F(k) C_{1}^{(l)}\right]^{T} P(k)\left[\sqrt{\tilde{\alpha}_{1}} B^{(l)} F(k) C_{1}^{(l)}\right] \\
& +\left[\sqrt{\tilde{\alpha}_{1}} F(k) C_{1}^{(l)}\right]^{T} R\left[\sqrt{\tilde{\alpha}_{1}} F(k) C_{1}^{(l)}\right] \\
& +\sum_{i=2}^{N}\left(\prod_{j=1}^{i-1} \sqrt{\left(1-\tilde{\alpha}_{j}\right) \tilde{\alpha}_{i}}\left[B^{(l)} F(k) C_{i}^{(l)}\right]^{T} P(k)\right. \\
& \left.\times \prod_{j=1}^{i-1} \sqrt{\left(1-\tilde{\alpha}_{j}\right) \tilde{\alpha}_{i}} B^{(l)} F(k) C_{i}^{(l)}\right) \\
& +\sum_{i=2}^{N}\left(\prod_{j=1}^{i-1} \sqrt{\left(1-\tilde{\alpha}_{j}\right) \tilde{\alpha}_{i}}\left[F(k) C_{i}^{(l)}\right]^{T} R\right. \\
& \left.\times \prod_{j=1}^{i-1} \sqrt{\left(1-\tilde{\alpha}_{j}\right) \tilde{\alpha}_{i}} F(k) C_{i}^{(l)}\right)+Q-P(k) \leq 0
\end{aligned}
$$

Pre- and post-multiplying (38) with $P^{-1}(k)$, then simplify the results with multiplying $\gamma(k)$, and let $W(k)=$ 
$\gamma(k) P^{-1}(k)$, we obtain:

$$
\begin{aligned}
& {\left[A^{(l)} W(k)+B^{(l)} F(k) \tilde{\Gamma}^{(l)} W(k)\right]^{T} W^{-1}(k)\left[A^{(l)} W(k)\right.} \\
& \left.+B^{(l)} F(k) \tilde{\Gamma}^{(l)} W(k)\right]+\left[\sqrt{\tilde{\alpha}_{1}} B^{(l)} F(k) C_{1}^{(l)} W(k)\right]^{T} \\
& \times W^{-1}(k)\left[\sqrt{\tilde{\alpha}_{1}} B^{(l)} F(k) C_{1}^{(l)} W(k)\right] \\
& +\left[\sqrt{\tilde{\alpha}_{1}} F(k) C_{1}^{(l)} W(k)\right]^{T} \gamma^{-1}(k) R\left[\sqrt{\tilde{\alpha}_{1}} F(k) C_{1}^{(l)} W(k)\right] \\
& +\sum_{i=2}^{N}\left(\prod_{j=1}^{i-1} \sqrt{\left(1-\tilde{\alpha}_{j}\right) \tilde{\alpha}_{i}}\left[B^{(l)} F(k) C_{i}^{(l)} W(k)\right]^{T} W^{-1}(k)\right. \\
& \left.\times \prod_{j=1}^{i-1} \sqrt{\left(1-\tilde{\alpha}_{j}\right) \tilde{\alpha}_{i}} B^{(l)} F(k) C_{i}^{(l)} W(k)\right) \\
& +\sum_{i=2}^{N}\left(\prod_{j=1}^{i-1} \sqrt{\left(1-\tilde{\alpha}_{j}\right) \tilde{\alpha}_{i}}\left[F(k) C_{i}^{(l)} W(k)\right]^{T} \gamma^{-1}(k) R\right. \\
& \left.\quad \times \prod_{j-1} \sqrt{\left(1-\tilde{\alpha}_{j}\right) \tilde{\alpha}_{i}} F(k) C_{i}^{(l)} W(k)\right) \\
& +W^{T}(k) \gamma^{-1}(k) Q W(k)-W(k) \leq 0 .
\end{aligned}
$$

By Schur complement lemma, and define $\tilde{W}(k) C_{s}^{(l)}=$ $C_{s}^{(l)} W(k)$ and $F(k) \tilde{W}(k)=Y(k)(s=1,2, \ldots, N, l=$ $1,2, \ldots, L),(39)$ is satisfied with conditions (14) and (11). Therefore, the proof is completed.

\section{REFERENCES}

[1] R.M. Alexandre, P.H. João, and N.N. Girish, Redundant data transmission in control/estimation over lossy networks, Automatica, vol. 48, no. 8, pp. 1612-1620, Aug. 2012.

[2] M. Canale, L. Fagiano and M. C. Signorile, Nonlinear model predictive control from data: a set membership approach, Int. J. Robust and Nonlin. Control, vol. 24, no. 1, pp. 123-139, Jan. 2014.

[3] M. Y. Chow, and Y. Tipsuwan, Gain adaption of networked DC motor controllers based on QoS variations, IEEE Trans. Ind. Electron., vol. 50, no. 5, pp. 936-943, Oct. 2003.

[4] B. Ding, Constrained robust model predictive control via parameterdependent dynamic output feedback, Automatica, vol. 46, no. 9, pp. 1517-1523, Sep. 2010.

[5] D. Ding, Z. Wang, B. Shen and H. Shu, $H_{\infty}$ state estimation for discrete-time complex networks with randomly occurring sensor saturations and randomly varying sensor delays, IEEE Trans. Neural Networks and Learning Syst. , vol. 23, no. 5, pp. 725-736, May 2012.

[6] D. Ding, Z. Wang, B. Shen and G. Wei, Event-triggered consensus control for discrete-time stochastic multi-agent systems: the input-tostate stability in probability, Automatica, Vol. 62, pp. 284-291, Dec. 2015.

[7] D. Ding, Z. Wang and B. Shen, Recent advances on distributed filtering for stochastic systems over sensor networks, International Journal of General Systems, Vol. 43, No. 3-4, pp. 372-386, 2014.

[8] D. Ding, Z. Wang, Fuad E. Alsaadi, and Bo Shen, Receding horizon filtering for a class of discrete time-varying nonlinear systems with multiple missing measurements, International Journal of General Systems, Vol. 44, No. 2, pp. 198-211, 2015.

[9] H. Dong, Z. Wang, F. E. Alsaadi and B. Ahmad, Event-triggered robust distributed state estimation for sensor networks with state-dependent noises, International Journal of General Systems, Vol. 44, No. 2, pp. 254-266, 2015.

[10] L.-S. Hu, B. Huang, and Y.-Y. Cao, Robust digital model predictive control for linear uncertain systems with saturations, IEEE Trans. Auto. Control, vol. 49, no. 5, pp. 792-796, May 2004.

[11] X. Ji, T. Liu, Y. Sun, and H. Su, Stability analysis and controller synthesis for discrete linear time-delay systems with state saturation nonlinearities, Int. J. Syst. Sci., vol. 42, no. 3, pp. 397-406, Mar. 2011.

[12] L. Jin, R. Kumar, and N. Elia, Model predictive control-based real-time power system protection schemes, IEEE Trans. Power Syst. , vol. 25, no. 2, pp. 988-997, May 2010.
[13] M.V. Kothare, V. Balakrishnan, and M. MorarI, Robust constrained model predictive control using linear matrix inequalities, Automatica, vol. 32, no. 10, pp. 1361-1379, Oct. 1996.

[14] M. Larsson and D. Karlsson, Coordinated system protection scheme against voltage collapse using heuritic search and predictive control, IEEE Trans. Power Syst., vol. 18, no. 3, pp. 1001-1006, Aug. 2003.

[15] D. Li, Y. Xi, and F. Gao, Synthesis of dynamic output feedback RMPC with saturated inputs, Automatica, vol. 49, no. 4, pp. 707-719, Apr. 2013.

[16] H. Li and Y. Shi, Event-triggered robust model predictive control of continuous-time nonlinear systems, Automatica, vol. 50, pp. 1507-1513, 2014.

[17] H. Li and Y. Shi, Robust distributed model predictive control of constrained continuous-time nonlinear systems: a robustness constraint approach, IEEE Trans. Auto. Control, vol. 59, no. 6, pp. 1673-1678, Jun 2014.

[18] J. Lu, D. Li, and Y. Xi, Constrained model predictive control synthesis for uncertain discrete-time Markovian jump linear systems, IET Controy Theory Appl., vol. 7, no. 5, pp. 707-719, Mar. 2013.

[19] D.Q. Mayne, Model predictive control: Recent developments and future promise, Automatica, vol. 50, no. 12, pp. 2967-2986, Dec. 2014.

[20] H. Nam, Y.C. Ko, and M.S. Alouini, Spectral efficiency enhancement in multi-channel systems using redundant transmission and diversity reception, IEEE Trans. Wireless Commun., vol. 7, no. 6, pp. 2143-2153, Jun. 2008.

[21] M.B. Shadmand, R.S. Balog, and H.A. Rub, Model predictive control of PV sources in a smart dc distribution system: maximum power point tracking and drop control, IEEE Trans. Energy Conversion, vol. 29, no. 4, pp. 913-921, Dec. 2014.

[22] Z. Wang, D. W. C. Ho, H. Dong, and H. Gao, Robust finite-horizon control for a class of stochastic nonlinear time-varying systems subject to sensor and actuator saturations, IEEE Trans. Autom. Control, vol. 55, no. 7, pp. 1716-1722, Jul. 2010 .

[23] F. Yang, Z. Wang, Y.S. Hung, and M. Gani, $H_{\infty}$ control for networked systems with random communication delays, IEEE Trans. Autom. Control, vol. 51, no. 3, pp. 511-518, Mar. 2006.

[24] R. Yang, G. Liu, P. Shi, C. Thomas, and M.V. Basin, Predictive output feedback control for networked control systems, IEEE Trans. Ind. Electron., vol. 61, no. 1, pp. 512-520, Jan. 2014.

[25] S. Yu, X. Li, H. Chen and F. Allgower, Nonlinear model predictive control for path following problems, Int. J. Robust and Nonlin. Control, vol. 25, no. 8, pp. 1168-1182, May 2015.

[26] L. Zhang, J. Wang, and C. Li, Distributed model predictive control for polytopic uncertain systems subject to actuator saturation, J. Process Control, vol. 23, no. 8, pp. 1075-1089, Sep. 2013.

[27] S. Zhang, and V. Vittal, Wide-area control resiliency using redundant communication paths, IEEE Trans. Power Syst. , vol. 29, no. 5, pp. 21892199, Sep. 2014.

[28] M. Zima, and G. Anderson, Model predictive control emloying trajectory sensitivities for power systems applications, in Proc. 44th IEEE Conf. Decision and Control, Seville, Spain, pp. 4452-4456, 2005.

[29] Z. Zou, Y. Bao, F. Deng, and H. Li, An approach of reliable data transmission with random redundancy for wireless sensors in structural health monitoring, IEEE J. Sensors, vol. 15, no. 2, pp. 809-818, Feb. 2015. 